\title{
Nivelación como Teoría Educativa para Carreras de Energías Renovables
}

\author{
Leveling as an Educational Theory for Renewable Energy Careers
}

Jose Antonio Hernández-Galvez', Rossana Aranda-Roche², Santiago Antonio Méndez $z^{3}$ y Verónika De la Cruz-Villegas ${ }^{4}$

\section{()} EDICIÓN: : 国IVTAC

Recibido: 11/julio/2020

Aceptado: 6/agosto/2020

Publicado: $25 /$ septiembre/2020

${ }^{1}$ México
${ }^{2}$ México
${ }^{3}$ México
${ }^{4}$ México

\section{IIIIInstitución}

1Universidad Juárez Autónoma de Tabasco ${ }^{2}$ Universidad Juárez Autónoma de Tabasco ${ }^{3}$ Universidad Juárez Autónoma de Tabasco ${ }^{4}$ Universidad Juárez Autónoma de Tabasco

\section{Correo Eletrónico}

1hernandezgalvezjoseantonio@gmail.com 2aranda_roche@yahoo.com 3santiagomendez@ujat.mx

4Veronika.delacruz@ujat.mx

\section{DORCID}

${ }^{1}$ https://orcid.org/0000-0003-3632-1490

${ }_{2}^{2}$ https://orcid.org/0000-0002-1971-7453

${ }^{3}$ https://orcid.org/0000-0002-3591-1168

${ }^{4} \mathrm{https}: / /$ orcid.org/0000-0003-3570-2021

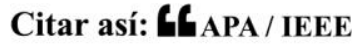

Hernández-Galvez, J., Aranda-Roche, R., Méndez, S. \& De la Cruz-Villegas, V. (2020). Nivelación como Teoría Educativa para Carreras de Energías Renovables. Revista Tecnológica-Educativa Docentes 2.0, 9(2), 95-102. https://doi.org/10.37843/rted.v9i2.150

\section{J. Hernández-Galvez, R. Aranda-Roche, S.} Méndez y V. De la Cruz-Villegas, "Nivelación como Teoría Educativa para Carreras de Energías Renovables", RTED, vol. 9, n. $^{\circ} 2$, pp. 95-102, sep. 2020.

\section{Resumen}

La física es una ciencia que brinda los conocimientos básicos que son necesarios para realizar estudios sobre energías renovables. El tener déficit en esta materia es una problemática generalizada en los estudiantes que ingresan al nivel universitario con el fin de estudiar dicha carrera. Pudo ser comprobado mediante pruebas diagnósticas, que el 57 $\%$ presentan dificultad con el concepto de energía, sus tipos, formas y transformaciones. Por dicha razón el objetivo buscado con este trabajo fue una propuesta didáctica que consiste en un curso de nivelación que se aplicó a 24 alumnos en la Universidad Popular de la Chontalpa, que forman un grupo piloto de primer año, primer semestre en esta carrera. Este curso se aplicó en el primer semestre de esta carrera. Se utilizó un método de investigación mixto que representó un conjunto de procesos sistemáticos, empíricos y críticos de investigación, que implican la recolección de datos tanto cuantitativo como cualitativo. Se llevó a cabo mediante la investigación acción y un estudio de caso basado en la propuesta de Stake (2005). Como resultado se observaron avances significativos en la interpretación del concepto de energía, su conservación, ejemplificar las fuentes renovables y explicaciones sobre las relaciones entre estas formas, mientras que se presentaron dificultades en las conversiones de unidades físicas. La propuesta didáctica contribuyó a mejorar las deficiencias en los alumnos y su desempeño; participando y realizando actividades durante las clases. Quedó demostrado que es necesario vincular elementos teóricos con la vida cotidiana y el trabajo colaborativo e individual.

Palabras clave: Propuesta didáctica\}, curso de nivelación, energías renovables, estrategias de aprendizaje.

\section{Abstract}

Physics is a science that provides the basic knowledge that is necessary to carry out studies on renewable energy. Having a deficit in this matter is a general problem in students who enter the university level to study said career. It could be verified through diagnostic tests that $57 \%$ have difficulty with the concept of energy, its types, forms, and transformations. For this reason, the objective sought with this work was a didactic proposal that consists of a leveling course that was applied to 24 students at the Popular University of Chontalpa, who form a first-year pilot group, first semester in this career. This course was applied in the first semester of this career. A mixed research method was used that represented a set of systematic, empirical, and critical research processes, which involve the collection of both quantitative and qualitative data. It was carried out through action research and a case study based on Stake's proposal (2005). As a result, significant advances were observed in the interpretation of the concept of energy, its conservation, exemplifying renewable sources and explanations about the relationships between these forms, while difficulties were presented in the conversions of physical units. The didactic proposal contributed to improve the deficiencies in the students and their performance, participating and doing activities during classes. It was demonstrated that it is necessary to link theoretical elements with daily life and collaborative and individual work.

Keywords: Didactic proposal; leveling course; renewable energy; learning strategies. 


\section{Introducción}

Los avances científicos y los progresos tecnológicos han surgido a partir de las propias necesidades del hombre. La ciencia, junto a las tecnologías son campos que crecen continuamente impulsados por nuevas inquietudes, curiosidades, problemas por resolver, o transformar. En este campo científico juega un importante papel estudiar física, tanto por sus desarrollos conceptuales y aplicaciones tecnológicas como por sus repercusiones sociales.

Por ello es necesario formar profesionistas proactivos, triunfadores, competentes, emprendedores; con competencia en el mercado laboral. Por otro lado, no se puede ignorar que la tecnología se ha desarrollado a un ritmo acelerado. Muchas veces en las clases hay alumnos que saben más informática que muchos maestros, pero sabe muy poca ciencia, en particular de las llamadas ciencias exactas. Esto nos obliga como profesionales a innovar, mejorar y si es necesario cambiar nuestra práctica docente.

Como plantea Vaillant (2013), las formas tradicionales de enseñar es necesario transformarlas, la sociedad y los estudiantes han cambiado, hay que cambiar nuestras prácticas docentes. En la actualidad resulta difícil enseñar ciencia, en particular física o alguna de sus especialidades. Los alumnos se preocupan más por discutir un fragmento observado en una película o una aplicación tecnológica, que un tema relacionado con las ciencias. Nuestros alumnos han ido perdiendo la curiosidad científica poco a poco en sus primeras etapas formativas. Los responsables de esta pérdida natural del ser humano son precisamente quienes practican didácticas ancestrales. Esta problemática surge porque la formación profesional didáctica- metodológica en muchos maestros se ha visto dañada, la cual lo limita a usar métodos, procedimientos y medios para enseñar adecuados.

Al respecto Zevallos (2020) resaltó el valor pedagógico de las Tecnologías del Aprendizaje y el Conocimiento (TAC) dentro del proceso de evaluación, aprendizajes. En dicho estudio también enfatizó en las necesidades formativas del profesional, así como implementar métodos para el aprendizaje tanto síncronos como asíncronos.

Usar la tecnología es una necesidad en la enseñanza de la física como ciencia; sin embargo, muchas instituciones educativas no cuentan con una dotación adecuada en los laboratorios de cómputo fundamentalmente en las secundarias básicas.

Esta misma situación se puede apreciar en los de ciencias, particularmente para el área de física, lo cual implica que no se puedan realizar las prácticas experimentales mínimas, por esta causa la mayoría del profesorado implementa clases teóricas, observándose el método tradicional, el uso del pizarrón como medio para enseñar de una manera adecuada.

Los diferentes trabajos realizados han demostrado que existe una problemática generalizada: los alumnos terminan la preparatoria y transitan por la universidad con déficit de conocimientos en algunas ciencias como física. Hechos fehacientes demostraron que le falta claridad para explicar conceptos, como energía, energía cinética, energía potencial gravitacional, solo por mencionar algunos; cuyos conceptos son importantes para aquellos alumnos al ingresan a carreras relacionadas con las energías renovables. Lo anterior implica una seria barrera en su formación como profesionista en esta área del conocimiento, ya que, al dificultársele una comprensión básica sobre dichos conceptos, les será difícil abordar sus cursos adecuadamente; egresando con serias deficiencias y sin poder complementar las competencias esperadas.

En los últimos años, los estudios han dado a conocer que los países comienzan a alarmarse por el analfabetismo científico, las bajas tasas de profesionistas en Ciencias, Tecnología, Ingeniería y Matemáticas (STEM por sus siglas en inglés). A ello está ligada la deserción escolar en los primeros semestres, dado entre otros factores por la mala calidad con la cual se imparten las clases, falta una infraestructura académica dirigida a resolver las necesidades existentes en los docentes y alumnos, hay una mala formación en la preparatoria afectando su rendimiento académico. Este hecho fue analizado por Vélez \& López (2007), quienes incluyen en su análisis factores como: estructura existente en la educación local, regional, nacional o mundial; la calidad en la formación del personal docente, las falsas expectativas creadas por la promoción. En este sentido el diseño de los programas de estudio tiene una importancia fundamental.

Algunos trabajos abordan dicha temática desde diferentes perspectivas. Por ejemplo, Montijo (2020), se basó en el modelo por competencias, realizó un análisis previo sobre las necesidades industriales para diseñar un programa que permitiera desarrollar acertadamente la materia "Diseño mecánico de 
precisión", en la carrera "Ingeniería Mecatrónica" del Instituto Tecnológico de Hermosillo, México. Ello le permitió incluir las competencias específicas y genéricas con mayor demanda a nivel local. Este es un aspecto que le da certidumbre a los futuros egresados, puesto que aumenta la probabilidad de que sean asimilados por el mercado laboral. En energías renovables los planes deben tomar esto en consideración, para que los contenidos en materia como física estén dirigidos a obtener el perfil, las competencias o aprendizaje esperados en los egresados, pero teniendo presente la existencia un problema real en los estudiantes; la carencia en sus conocimientos básicos sobre el concepto de energía.

Esta situación ha provocado un mayor interés en la comunidad científica hacia la enseñanza de la física, ha mantenido preocupado y ocupado a investigadores en diferentes países. Según Calvo (2005), los problemas asociados a la enseñanza en esta materia se han presentado desde la secundaria básica, donde los contenidos, así como el tiempo dedicado a las clases en el curso han sufrido una enorme reducción en las sucesivas reformas educativa. Mientras que, Castiblanco (2008), Alcivar y Chiquitico (2007) se han enfocado en el uso que tienen las Tecnologías de la Información y la Comunicación (TIC) para enseñar física, debido a las múltiples oportunidades ofrecidas. Desarrollar laboratorios virtuales, simular ciertos fenómenos físicos mediante modelos matemáticos, solo por mencionar algunos algunas, además brinda la posibilidad para realizar trabajos colaborativos.

El uso de las TIC ya había sido sugerido por Arrieta y Delgado (2006) para enseñar física en el nivel básico. Ellos demostraron que es necesario formar previamente a los profesores, ya que ellos en un alto por ciento tienen dificultad para usarla. A de más Lara (2010), desarrolló una estrategia didáctica para enseñar física, fundamentada en la interpretación de cocientes. Por otra parte, Quirantes (2011) propuso una herramienta docente con la cual se puede enseñar física en el nivel universitario usando fragmentos de películas, lo cual constituye una buena alternativa para enseñar física desde otra mirada. El método contribuyó a comprender mejor ciertos fenómenos físicos, así como a retener las ideas en los estudiantes que estudiaban Química en la universidad granadiense.

Un trabajo similar fue realizado por Rincón (2020), al aplicar la teoría cognitiva de Jerome Bruner para fortalecer en los estudiantes su conducta hacia la protección del medioambiente.
Dicho estudio tuvo un carácter cualitativo, se apoyó en el método investigación-acción. La estrategia didáctica incluyó elementos lúdicos, musicales, teatrales poéticos; cuya efectividad quedó demostrada a través del dinamismo en las actividades realizadas, la capacidad desarrollada en los estudiantes para hacer análisis. En este sentido Sterna, Echeverría y Porta (2017) desarrollaron un método para la enseñanza de la física basado en experimentos, donde se relacionan los procesos físicos con la vida real. El trabajo experimental les permitió a los estudiantes universitarios integrar y construir conocimientos mediante el trabajo colectivo, aumentando continuamente la participación.

En cuanto a las investigaciones realizadas y publicadas en la enseñanza de la física como base para la energía renovable, se puede decir que no abundan en la literatura. Torres, Rincón, Lentz y González (2013) utilizaron un calentador solar de agua para explicar cómo tienen lugar en él ciertos procesos físicos. Su estrategia para enseñar física se apoyó en archivos multimedia como fotos, videos y animaciones por computadora. Por otro lado, las investigaciones de Gómez et al. (2020) permitieron demostrar la efectividad del método del aula invertida para enseñar física en estudiantes que se dedican a las ingenierías. Dicho método fue utilizado satisfactoriamente para llevar a cabo prácticas de laboratorio, dónde los estudiantes reciben materiales con anterioridad para su estudio.

También se han hecho algunos estudios para el nivel de secundaria básica donde se diseñan los materiales curriculares (Pérez y Varela, 2006). El estudio comenzó realizando un análisis en cuanto a los conocimientos previos relacionados con la energía; estando enfocado en la energía eléctrica, interna y mecánica. Enseñar física es un desafío al cual nos enfrentamos continuamente los profesores del área, en este sentido juegan un importante papel las instituciones educativas en sus diferentes niveles. Moreira (2014) realizó un análisis de los principales desafíos que tiene esta enseñanza en la educación contemporánea, entre los cuales citó los siguientes: siempre se debe considerar el conocimiento previo, debido a su gran influencia sobre los nuevos por aprender; presentar a los alumnos situaciones correspondientes a su entorno, edad, cultura; la enseñanza no debe ser monológica, sino dialógica.

Pese a los esfuerzos que se han realizado en la 
enseñanza de la física en diferentes partes del mundo, las dificultades continúan presentándose. En México en particular, son pocas las investigaciones realizadas en esta área. Por ello, este trabajo pretende atender dicha problemática, mediante un curso de nivelación, el cual retomará aquellos contenidos relacionados con el concepto de energía estudiado en la secundaria básica o preparatoria, ya que son necesarios para los que estudian la carrera de energía renovable en la universidad. Esta propuesta de nivelación se hizo con el fin de llevarla a cabo en la Universidad Popular de la Chontalpa en un grupo de 24 alumnos, del primer año de la carrera "Energías Renovables". Nivelar estos contenidos en el primer semestre del primer año de la carrera facilitará a los alumnos su tránsito por el curso sin dificultad.

\section{Metodología}

El trabajo desarrollado es un estudio de caso llevado a cabo en la Universidad Popular de la Chontalpa, en un grupo de 24 alumnos que se especializan en energía renovable. Este estudio de caso está basado en la propuesta de Stake (2005), el cual define un estudio de caso como una investigación particularmente apropiada para estudiar un caso o situación con cierta intensidad en cierto periodo y pueden clasificarse por la naturaleza del informe final. Así Merriam (1998) los agrupa en tres tipologías diferentes: descriptivos, interpretativos y evaluativos.

Dadas las características de esta investigación, se realizará un estudio con carácter evaluativo. En este sentido el método investigativo es propio ya que se buscó realizar un diagnóstico en un grupo específico y luego de tener los resultados, así como los ejes problematizadores, ser sometidos a una intervención, delimitando la problemática y a los sujetos seleccionados al inicio del proyecto.

En lo referente al método de investigación utilizado, el mismo fue mixto (cualitativo y cuantitativo). De acuerdo con (Hernández y Mendoza, 2008) este método representa un conjunto de procesos sistemáticos, empíricos y críticos de investigación, que implican la recolección de datos tanto cuantitativos como cualitativos. Estos métodos se pueden integrar para realizar una discusión conjunta de toda la información recabada, logrando un mayor entendimiento del fenómeno bajo estudio.

Estos métodos se combinan, al menos un componente cuantitativo y uno cualitativo en un mismo estudio o proyecto de investigación, es decir facilitan tener una visualización más amplia de la investigación (Chen, 2006; Johnsonetal, 2006). Por otro lado, se pueden mezclar ambos enfoques (Johnson et al. 2006) enfatizando más en uno de ellos o dándole la misma importancia a los dos. Es necesario señalar que cuando se hable del método cuantitativo éste se abreviará como CUAN y cuando se trate del método cualitativo como CUAL).

Utilizar los métodos mixtos permite lograr una perspectiva más amplia y profunda del fenómeno, la investigación se sustenta en las fortalezas de cada método y no en sus debilidades potenciales. Todd, Nerlich y McKeown (2004) señalan que con el enfoque mixto se exploran distintos niveles del problema de estudio. Se puede Formular el planteamiento del problema con mayor claridad, así como las maneras más apropiadas para teorizarlo (Brannen, 1992).

Además, se utilizó la investigación-acción, la cual es un diseño metodológico que permite realizar investigaciones e intervenciones, mismas que Pérez (1998) menciona como un proceso activo, sistemático y riguroso de indagación dirigida, en la que se toman decisiones sobre lo que se investiga, estando en el campo que se estudia. Se usó este diseño porque se pretende retomar los déficits cognitivos que tienen los egresados de la preparatoria y que ingresan a la universidad a estudiar energía renovable con relación al concepto de energía y sus implicaciones.

Esta investigación, nos ayuda a establecer un proceso de mejora continua mediante un curso de nivelación llevado a cabo en el primer semestre de la carrera en la universidad ya mencionada, con la participación de todos los involucrados y que en este tránsito todos conformen una comunidad para lograr un aprendizaje significativo La investigación-acción es una herramienta que permite resolver problemas, a partir del momento en el que se ha entendido y comprendido una realidad socioeducativa. Para Kemmis (citado en Latorre, 2005), la investigaciónacción, es más que un proceso riguroso de investigación o una ciencia práctica y moral, es también un proceso que facilita realizar una investigación crítica. Para este autor la investigación acción es:

Una forma de indagación autor reflexiva realizada por quienes participan (profesorado, alumnado o dirección, por ejemplo) en las situaciones 
sociales (incluyendo las educativas) para mejorar la racionalidad y la justicia de: a) sus propias prácticas sociales o educativas; b) su comprensión sobre las mismas; y c) las situaciones e instituciones en que estas prácticas se realizan. La investigación - acción supone entender la enseñanza como un proceso de investigación de continua búsqueda. Esta investigación proporciona herramientas muy útiles que contribuyen a darle tratamiento a la problemática identificada. A la vez, permite evaluar los resultados obtenidos y realizar la retroalimentación necesaria".

Para determinar la metodología y estructura del proyecto, se consideró lo planteado en el manual para el desarrollo del trabajo terminal de la Maestría en Intervención e Innovación de la Práctica Educativa (MIIPE). Para su implementación se tuvieron en cuenta cuatro etapas: identificación del problema; diagnóstico; propuesta; ejecución y evaluación. Para identificar el problema se tuvo en cuenta la experiencia docente acumulada por los autores del trabajo, los profesores del Cuerpo Académico "Energía y Medioambiente" radicado en la UPCH, en el área de las ciencias básicas, especialmente en física. Se implementó un análisis cualitativo para darle tratamiento a dicho concepto.

El diagnóstico se realizó a un grupo piloto de 24 alumnos egresados de la preparatoria y que están estudiando energías renovables en la universidad, para conocer las dificultades con las cuales entran relacionadas con el concepto de energía. Según el diccionario reverso (2020) un grupo piloto es un pequeño conjunto de personas reunidas, con rasgos comunes. Se identificaron los problemas existentes, teniendo en cuenta la variable académica centrada en el alumno, para obtener los ejes problematizadores que luego fueron atendidos durante la aplicación del proyecto.

Los ejes identificados fueron: es insuficiente la interpretación realizada sobre el concepto de energía; tienen dificultad en el trabajo algebraico para darle solución a los problemas físicos; no se han apropiado del procedimiento a seguir para la convertir magnitudes físicas usada cotidianamente, la misma le serán importante en su carrera. En la recolección de datos se utilizó como instrumento un cuestionario a 24 alumnos de la carrera (validado en base a una prueba piloto) para identificar sus deficiencias.

De acuerdo con Ucha (2014) una prueba piloto es el nombre con el cual se denomina a aquella prueba iniciática, es decir, que se lleva a cabo por primera vez y que se desarrolla a escala pequeña con un fin experimental, para poder comprobar si determinadas situaciones son viables o no. Esta prueba permitió conocer si los alumnos sabían interpretar el concepto de energía, el principio de conservación y trasformación, así como ejemplificar fuentes renovables de energía, explicar las magnitudes involucradas en la energía cinética, potencial, interpretar resultados físicos, convertir magnitudes y trabajo algebraico.

Una vez conocidos los resultados del diagnóstico y los ejes problematizadores, se realizó una propuesta didáctica que consistió en un curso de nivelación para atender las dificultades identificadas. Este curso fue diseñado para desarrollarse en el primer semestre y año de la carrera en energías renovables. El plan de acción desarrollado para su ejecución se llevó a cabo en tres sesiones, como se puede observar en la tabla 1. Los contenidos en cada sesión se formularon teniendo en cuenta los resultados del diagnóstico previamente realizado. Se resolvieron ejercicios donde debían realizar despejes, convertir magnitudes, trabajo algebraico e interpretar resultados físicos.

\section{Tabla 1}

Distribución del contenido por sesiones de clases. Sesiones y su duración en horas/clase $(\mathrm{h} / \mathrm{c})$

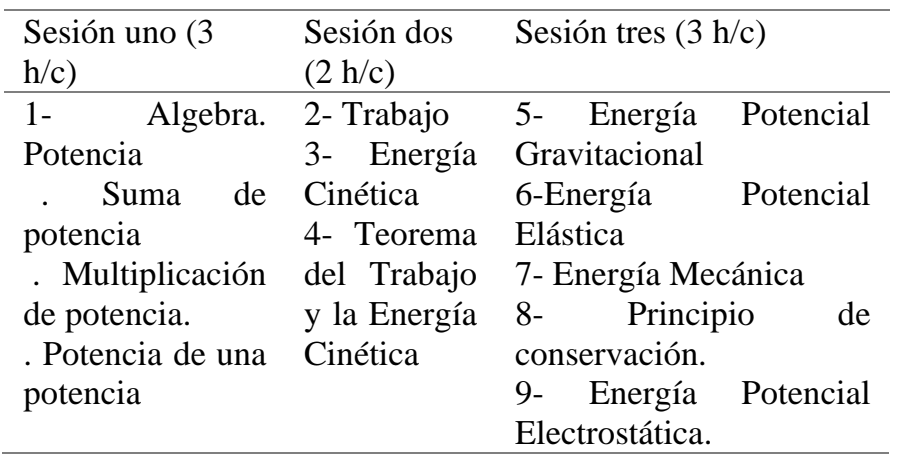

Nota. Los contenidos de las secciones respondieron a las dificultades identificadas y a los ejes problematizadores, elaboración propia.

La sesión uno se dedicó al trabajo algebraico, en especial al cálculo de potencia en sus diversas formas. Su desarrollo se hizo retomando contenidos previos mediante preguntas, dirigiendo el debate sobre el concepto de potencia; seguidamente se realizó un análisis sobre cómo resolver ejercicios relacionados el mismo. Para darle cumplimiento a la metodología empleada se trabajó en equipos, estos no debían tener más de cuatro alumnos para trabajar 
colaborativamente, lo cual ayudó mucho a la apropiación del algoritmo usado para trabajar. Las dos siguientes sesiones se dedicaron a trabajar los temas correspondientes al concepto de energía y las implicaciones que este tiene en las energías renovables. Se realizó un análisis de este concepto, su ecuación matemática, magnitudes involucradas y unidades utilizadas para su medición.

La etapa de evaluación permitió evaluar el impacto que tuvo el curso, para lo cual se elaboró un examen como instrumento mediante un cuestionario (validado con base a una prueba piloto), que nos permitiera conocer los avances obtenidos por los estudiantes, relacionados con dicho concepto, con sus implicaciones. Se evaluaron cinco reactivos, con preguntas abiertas y cerradas, tuvo un tiempo de duración de dos horas. En las preguntas abiertas debían solucionar problemas, realizar trabajo algebraico, conversión de magnitudes. En las preguntas cerradas solo debían interpretar conceptos mediante situaciones dadas; además, se llevó a cabo una evaluación acumulativa durante todo el curso. En. Se seleccionó la técnica de la encuesta a través de un cuestionario como instrumento, conformado por seis ítems con una escala tipo Likert llevado de uno a cinco donde uno era el menor grado de satisfacción y cinco el mayor.

\section{Resultados}

A continuación, se describen los resultados fundamentales que fueron alcanzados con el desarrollo del estudio. El análisis realizado permite medir los avances que experimentaron los estudiantes una vez implementado el curso de nivelación, así como identificar los aspectos conceptuales en los que menos se logró una comprensión adecuada por parte de estos.

En la tabla 2 podemos observar que, al evaluar la interpretación del concepto de energía, en el diagnóstico inicial reprobaron 12 alumnos (50\%), mientras que después solo reprobaron 5 (28.83\%). En la interpretación del principio de conservación, los resultados fueron mejores, solo reprobaron 4 alumnos (16.67\%) en el diagnóstico inicial y cuando se aplicó el curso no reprobó ninguno; lográndose un $100 \%$. En cuanto al objetivo donde debían ejemplificar fuentes renovables de energía se pudo observar que, en un inicio reprobaron 9 alumnos $(37.50 \%)$, después que se aplicó la nivelación la aplicación reprobó 3 (12.50\%); sin embargo, cuando fueron a explicar la relación que existe entre la energía potencial y las magnitudes involucradas, inicialmente reprobaron 14 alumnos $(58.3 \%)$ y luego reprobaron $6(25 \%)$.

Tabla 2

Resultado de la aplicación del curso de nivelación.

\begin{tabular}{|c|c|c|c|c|c|c|c|c|}
\hline \multirow[t]{2}{*}{$\begin{array}{l}\text { Objetivos } \\
\text { evaluados }\end{array}$} & \multicolumn{4}{|c|}{$\begin{array}{c}\text { Diagnóstico } \\
\text { inicial }\end{array}$} & \multicolumn{4}{|c|}{ Diagnóstico final } \\
\hline & $\mathrm{R}$ & $\%$ & A & $\%$ & $\mathrm{R}$ & $\%$ & A & $\%$ \\
\hline $\begin{array}{l}\text { 1- Interpretar el } \\
\text { concepto de } \\
\text { energía }\end{array}$ & $\begin{array}{l}1 \\
2\end{array}$ & 50 & $\begin{array}{l}1 \\
2\end{array}$ & 50 & 5 & $\begin{array}{l}20 . \\
83\end{array}$ & $\begin{array}{l}1 \\
9\end{array}$ & $\begin{array}{l}79 . \\
17\end{array}$ \\
\hline $\begin{array}{l}\text { 2- Interpretar el } \\
\text { principio de } \\
\text { conservación de } \\
\text { la energía }\end{array}$ & 4 & $\begin{array}{l}16 . \\
67\end{array}$ & $\begin{array}{l}2 \\
0\end{array}$ & $\begin{array}{l}83 . \\
33\end{array}$ & 0 & $\begin{array}{c}0.0 \\
0\end{array}$ & $\begin{array}{l}2 \\
4\end{array}$ & $\begin{array}{l}100 \\
.00\end{array}$ \\
\hline $\begin{array}{l}\text { 3- Ejemplificar } \\
\text { fuentes } \\
\text { renovables de } \\
\text { Energía }\end{array}$ & 9 & $\begin{array}{l}37 . \\
50\end{array}$ & $\begin{array}{l}1 \\
5\end{array}$ & $\begin{array}{l}62 . \\
50\end{array}$ & 3 & $\begin{array}{l}12 . \\
50\end{array}$ & $\begin{array}{l}2 \\
1\end{array}$ & $\begin{array}{l}87 . \\
50\end{array}$ \\
\hline $\begin{array}{l}\text { 4- Explicar de } \\
\text { qué depende la } \\
\text { energía potencial } \\
\text { y la cinética }\end{array}$ & $\begin{array}{l}1 \\
4\end{array}$ & $\begin{array}{l}58 . \\
33\end{array}$ & $\begin{array}{l}1 \\
0\end{array}$ & $\begin{array}{l}41 . \\
67\end{array}$ & 6 & $\begin{array}{l}25 . \\
00\end{array}$ & $\begin{array}{l}1 \\
8\end{array}$ & $\begin{array}{l}75 . \\
00\end{array}$ \\
\hline $\begin{array}{l}\text { 5- Realizar } \\
\text { trabajo } \\
\text { algebraico }\end{array}$ & $\begin{array}{l}1 \\
8\end{array}$ & 75 & 6 & 25 & 8 & $\begin{array}{l}33 . \\
33\end{array}$ & $\begin{array}{l}1 \\
6\end{array}$ & $\begin{array}{l}66 . \\
67\end{array}$ \\
\hline $\begin{array}{l}\text { 6- Interpretar } \\
\text { resultados físicos }\end{array}$ & $\begin{array}{l}1 \\
4\end{array}$ & $\begin{array}{l}58 . \\
33\end{array}$ & $\begin{array}{l}1 \\
0\end{array}$ & $\begin{array}{l}41 . \\
67\end{array}$ & 9 & $\begin{array}{l}37 . \\
50\end{array}$ & $\begin{array}{l}1 \\
5\end{array}$ & $\begin{array}{l}62 . \\
50\end{array}$ \\
\hline $\begin{array}{l}\text { 7- Convertir } \\
\text { magnitudes }\end{array}$ & $\begin{array}{l}2 \\
1\end{array}$ & $\begin{array}{l}87 . \\
50\end{array}$ & 3 & $\begin{array}{l}12 . \\
50\end{array}$ & $\begin{array}{l}1 \\
3\end{array}$ & $\begin{array}{l}53 . \\
17\end{array}$ & $\begin{array}{l}1 \\
1\end{array}$ & $\begin{array}{l}45 . \\
83\end{array}$ \\
\hline
\end{tabular}

Nota. Indicadores R: Reprobados; A: Aprobados, elaboración propia.

Uno de los problemas actuales en los estudiantes es el trabajo algebraico. Al evaluar este objetivo se pudo comprobar que en el diagnóstico inicial reprobaron $18(75 \%)$, en el final reprobaron 8 $(33.33 \%)$, este es un tema pendiente en el cual es necesario seguir trabajando sistemática. Para estudiar las energías renovables es importante saber interpretar un resultado físico. En este objetivo se observó en el diagnóstico inicial hubieron14 estudiantes reprobados $(58.33 \%)$, luego reprobaron $9(37 \%)$. En la vida diaria interactuamos muchas veces inconscientemente con diferentes magnitudes físicas expresadas en determinada unidad de medida, su conversión en otra unidad reviste gran importancia. Este fue otro objetivo donde se presentó serias dificultades. En el diagnóstico inicial reprobaron $21(87.50 \%)$, luego de aplicar el curso reprobaron 13 (53.17\%).

Para darle cumplimiento a la metodología 
investigación acción, durante la implementación se priorizó el trabajo del alumno, primero en equipos, luego individualmente, buscando desarrollar en ellos cierta independencia cognitiva y pudieran por sí solo resolver los ejercicios propuestos. Luego se le dio la posibilidad de proponer otros ejercicios similares a los analizados. Esta dinámica llevada a cabo fue fundamental en los avances logrados, los cuales mostramos en la tabla 3. El avance obtenido por los alumnos en cada objetivo se realizó teniendo en cuenta los reprobados en el diagnóstico inicial. En la interpretación del concepto de energía reprobaron inicialmente 12 alumnos, solo reprobaron 5; lográndose que 7 alumnos avanzaran, representando un $58.33 \%$.

\section{Tabla 3}

Avances con relación al diagnóstico inicial.

\begin{tabular}{|c|c|c|c|}
\hline \multirow{2}{*}{$\begin{array}{l}\text { Objetivos } \\
\text { evaluados }\end{array}$} & \multicolumn{3}{|c|}{ Alumnos Reprobados } \\
\hline & $\begin{array}{l}\text { Diagnóstico } \\
\text { inicial } \\
\text { (cantidad) }\end{array}$ & $\begin{array}{l}\text { Diagnóstico } \\
\text { final } \\
\text { (cantidad) }\end{array}$ & $\begin{array}{c}\text { Avances } \\
(\%)\end{array}$ \\
\hline $\begin{array}{l}\text { 1- Interpretar el } \\
\text { concepto de } \\
\text { Energía }\end{array}$ & 12 & 5 & 58.33 \\
\hline $\begin{array}{ll}\text { 2- Interpretar el } \\
\text { principio } & \text { de } \\
\text { conservación } & \text { de } \\
\text { la Energía } & \end{array}$ & 4 & 0 & 100.00 \\
\hline $\begin{array}{l}\text { 3- Ejemplificar } \\
\text { fuentes } \\
\text { renovables de } \\
\text { Energía }\end{array}$ & 9 & 3 & 66.67 \\
\hline $\begin{array}{l}\text { 4- Explicar de qué } \\
\text { depende la } \\
\text { Energía potencial } \\
\text { y la cinética }\end{array}$ & 14 & 6 & 57.14 \\
\hline $\begin{array}{l}5-\quad \text { Realizar } \\
\text { trabajo } \\
\text { algebraico }\end{array}$ & 18 & 8 & 55.56 \\
\hline $\begin{array}{l}\text { 6- Interpretar } \\
\text { resultados físicos }\end{array}$ & 14 & 9 & 35.71 \\
\hline $\begin{array}{l}\text { 7- Convertir } \\
\text { magnitudes } \\
\text { físicas }\end{array}$ & 21 & 13 & 38.10 \\
\hline
\end{tabular}

Nota. Diagnóstico inicial aprobaron, elaboración propia.

En el principio de conservación los 4 alumnos reprobados en el diagnóstico inicial aprobaron luego que se aplicó la propuesta didáctica; en este objetivo el avance fue de un $100 \%$. Al ejemplificar fuentes renovables de energía había 9 alumnos reprobados, sin embargo, cuando se volvió a evaluar este objetivo solo reprobaron 6, aprobando 8 alumnos con un avance del
$66.67 \%$

$\mathrm{Al}$ evaluar la dependencia que tienen la energía cinética y la potencial con relación a las magnitudes involucradas en sus ecuaciones, en un inicio había 14 alumnos reprobados y avanzaron 8, el $57.14 \%$. En cuanto al trabajo algebraico los resultados no fueron los mejores, aún quedan problemas por resolver. En el diagnóstico inicial reprobaron 18 alumnos, luego de aplicar la propuesta de nivelación reprobaron 8 , logrando avanzar solo 10 alumnos representando un $55.56 \%$.

Los resultados obtenidos en la interpretación de resultados físicos no fueron los esperados, inicialmente reprobaron 14 alumnos, luego de aplicase el curso reprobaron 9, con un avance del $35.71 \%$. Mientras que, en las conversiones físicas inicialmente reprobaron 21, luego del curso reprobaron 13, logrando avanzar el $38.10 \%$. Con relación a la encuesta aplicada para conocer el grado de satisfacción que tuvieron los alumnos con el curso recibido, el $41.6 \%$ estuvo satisfecho y 12 alumnos tuvieron por debajo del grado más alto de satisfacción.

Nos interesó conocer si los contenidos del curso fueron útiles para ellos. El $83.3 \%$ encontró utilidad en los contenidos seleccionados; solo 4 alumnos no estuvieron satisfechos. Al evaluar si se lograron los objetivos propuestos, el $58.3 \%$ estuvo satisfecho. Además, durante el desarrollo del curso se utilizaron materiales suficientes. Al preocuparnos por este aspecto el $58.3 \%$ estuvo satisfecho. Cuando preguntamos si las actividades fueron adecuadas con relación a las temáticas abordadas, el $87.5 \%$ estuvo de acuerdo. Al preguntar si las sesiones trabajadas fueron suficientes, el $54.16 \%$ dijo que era necesario utilizar un número mayor de sesiones.

\section{Conclusiones}

Los alumnos que estudian ingeniería en energía renovable en la Universidad Popular de la Chontalpa debían conocer a un nivel básico, los conceptos relacionados con la energía y sus implicaciones. Sin embargo, a partir del diagnóstico aplicado a un grupo piloto se pudo comprobar la falta de estos conceptos en los estudiantes, tampoco dominaban con claridad las operaciones algebraicas necesarias para manejar el cálculo en los problemas físicos. Esto les impide llevar en forma adecuada los cursos del primer año en su formación profesional adecuadamente. 
La propuesta didáctica desarrollada permitió comprobar que la dinámica llevada a cabo para trabajar mediante una retroalimentación continua contribuyó a mejorar las deficiencias existentes en los alumnos, su desempeño, participación y realización de actividades durante las clases, por lo que ha sido especialmente beneficioso en los estudiantes de ingeniería en energía renovables.

Lo anterior estuvo apoyado por una adecuada vinculación del contenido con la vida cotidiana, este hecho estimuló la participación en las clases facilitando la ejecución del proyecto. Esta propuesta didáctica sirvió como iniciativa para implementarla en próximos cursos de esta carrera, teniendo en cuenta la importancia de los temas impartidos. Otro elemento concluyente es la necesidad que tienen las instituciones educativas, en especial la Universidad Popular de la Chontalpa, adquirir equipamientos para los laboratorios que les permita a los profesores dar clases demostrativas y realizar prácticas experimentales.

Las teorías, los conceptos físicos, no se comprenden adecuadamente si solo se basan en clases teóricas, este hecho es aún más difícil para los que estudian energía renovable pues carecen de esta formación conceptual básica, siendo aún más complicada para desarrollar estudios en otras carreras. Los recursos financieros en las instituciones educativas son escasos lo cual nos hace pensar en la necesidad de aplicar esta propuesta didáctica.

\section{Reconocimiento}

Se reconoce el apoyo brindado por el Cuerpo Académico "Energía y Medioambiente", integrado por los profesores Dr. Omar Sarracino Martínez, Dr. Geovanni Hernández Galvez y M.C. Antonio Trujillo Narcia. También se agradece a la segunda generación de estudiantes que estudian energías renovables en la UPCH, por su participación.

\section{Referencias}

Arrieta, X. y Delgado, M. (2006). Tecnologías de la información en la enseñanza de la física de educación básica. Enlace, 3(1), 63-76. Recuperado de: http://ve.scielo.org/scielo.php?script=sci_arttext $\&$ pid=S1690$75152006000100005 \& \operatorname{lng}=$ es\&tlng=es.

Calvo, A. (2005). Nuevos enfoques para la enseñanza de la física. Instituto de Formación de Profesores. Recuperado de: https://sede.educacion.gob.es/publiventa/PdfServlet?pdf=VP1 2026.pdf\&area $=\mathrm{E}$
Castiblanco, O. (2008). El uso de las TIC en la enseñanza de la Física. Recuperado http://www.unilibre.edu.co/revistaingeniolibre/revista7/articulos/Eluso-de-las-TICs.pdf.

Gómez J.A., Vidaurre A., Tort I., Molina J., Serrano M.A., Meseguer J.M., Martínez R.M, Quiles S. y Riera J. (2020). Effectiveness of flip teaching on engineering students' performance in the physics lab. Computers \& Education $144 \quad$ (103708). https://doi.org/10.1016/j.compedu.2019.103708.

Lara, A. (2010). Interpretación de cociente. Revista Eureka sobre Enseñanza y Divulgación de las Ciencias. Recuperado de http://www.apaceureka.org/revista

Loor, B.J, Chiquito, S.L., y Rodríguez, S.M. (2017). Las TIC en el aprendizaje de la Física. Revista Publicando, 4 (1). 429-438.

Montijo, E. (2020). Generación del Programa de Estudios "Diseño Mecatrónico de Precisión" del Instituto Tecnológico de Hermosillo, Bajo el Modelo Curricular Basado en Competencias. Revista Tecnológica-Educativa Docentes 2.0, 9(1), 56-62. https://doi.org/10.37843/rted.v9i1.108.

Moreira, M.A. (2014). Enseñanza de la física: aprendizaje significativo, aprendizaje mecánico y criticidad. Revista de Enseñanza de la Física. Vol. 26, No. 1, dic. 2014, 45-52 https://revistas.unc.edu.ar/index.php/revistaEF/article/view/9515/1029 0 .

Pérez, M.C. y Varela, M.P. (2006). Una propuesta para desarrollar en el alumno de secundaria una visión unificada de la física a partir de la energía. Revista Eureka sobre Enseñanza y Divulgación de las Ciencias, vol. 3 , núm. 2, 2006, pp. 237-250. Consultado el 15 de julio del 2020. http://www.redalyc.org/articulo.oa?id=92030206.

Quirantes, A. (2011). Física de Película: una herramienta docente para la enseñanza de Física universitaria usando fragmentos de películas. Revista Eureka Sobre Enseñanza Y Divulgación De Las Ciencias, 8(3), pp. 334-340. Consultado el 15 de junio del 2020. https://revistas.uca.es/index.php/eureka/article/view/2721.

Rincón, F. (2020). Análisis de la aplicación de la teoría cognitiva de Jerome Bruner como mecanismo para fortalecer la conducta ambiental en los estudiantes del grado segundo de la institución educativa Chuniza. Revista Tecnológica-Educativa Docentes 2.0, 9(1), 132-141. https://doi.org/10.37843/rted.v9i1.110.

Stern C., Echeverría C. y Porta D. (2017). Teaching physics through experimental projects. Procedia IUTAM 20, 189-194. https://doi.org/10.1016/j.piutam.2017.03.026.

Torres M. J, Rincón M.E., Lentz H. A. y González C. L. (2013). Alternative Energies in Physics, a Proposal for Exploring the Teaching of Physics Concepts with the Solar Water Heater. Energy Procedia 57, 975 - 981. doi: 10.1016/j.egypro.2014.10.080.

Vaillant, D. (2013). Integración de TIC en los sistemas de formación docente inicial y continua para la Educación Básica en América Latina: Argentina. Recuperado de: https://www.researchgate.net/publication/256487100_Integracion_de_ TIC_en_los_sistemas_de_formacion_docente_inicial_y_continua_para _la_Educacion_Basica_en_America_Latina.

Vélez A. y López D.F. (2007). Estrategias para vencer la deserción universitaria. Educación y Educadores, Volumen 7, 177-203. Consultado el 14 de julio del 2020. http://dspace.uhemisferios.edu.ec:8080/xmlui/handle/123456789/405? show=full.

Zeballos, M. (2020). La evaluación de los aprendizajes mediadas por las TAC. Revista Tecnológica-Educativa Docentes 2.0, 9(1), 83-95. https://doi.org/10.37843/rted.v9i1.98. 